\title{
fur Gene Expression as a Discriminating Marker for Small Cell and Nonsmall Cell Lung Carcinomas
}

\author{
Jack A. Schalken," Anton J. M. Roebroek, ${ }^{\ddagger}$ Paul P. C. A. Oomen, ${ }^{5}$ Sjoerd Sc. Wagenaar," Frans M. J. Debruyne," \\ Henri P. J. Bloemers, ${ }^{\ddagger}$ and Wim J. M. Van de Ven ${ }^{\ddagger}$ \\ ${ }^{*}$ Department of Urology, University of Nijmegen, Geert Grooteplein Zuid 16, 6500 HB Nijmegen, The Netherlands; ${ }^{\ddagger}$ Molecular \\ Oncology Section, Department of Biochemistry, University of Nijmegen, Geert Grooteplein Noord 21, 6525 EZ Nijmegen, \\ The Netherlands; ${ }^{\S}$ Department of Physiological Chemistry, State University of Utrecht, Utrecht, The Netherlands; \\ and "Department of Pathology, St. Antonius Hospital, Nieuwegein, The Netherlands
}

\begin{abstract}
The recently discovered fur gene encodes a membrane-associated protein with a recognition function. To further characterize the gene, we studied its expression by Northern blot analysis using poly(A)-selected RNA from a variety of organs of African green monkey and rat. The fur gene appeared to be differentially expressed, relatively high levels of fur mRNA being present in specimens of liver and kidney, low levels in brain, spleen, and thymus, and very low levels in heart muscle, lung, and testis. mRNA levels in specimens of human lung tissue without neoplastic lesions were also very low. Similar analyses of primary human lung carcinomas of different histopathological types revealed a highly selective and strong elevation of fur expression in nonsmall cell lung carcinomas, but not in small cell lung carcinomas. These results indicate that fur expression can be used to discriminate between these two types of human lung cancer.
\end{abstract}

\section{Introduction}

Recently, we have described a new gene that we designated fur (1). The gene was discovered when the regions immediately upstream of the human and feline fes/fps proto-oncogenes were compared by Southern blot and heteroduplex analysis in an attempt to define more precisely the $5^{\prime}$ ends of these protooncogenes. The fur transcription unit appeared to be distributed over a DNA region of $\sim 10 \mathrm{kbp}$ and to code for an mRNA of $4.5 \mathrm{~kb}$. The poly(A) addition site of the fur messenger was found in very close proximity of the $f e s / f p s$ proto-oncogene (1).

Nucleotide sequence analysis of fur-related cDNA clones showed that the $3^{\prime}$ end of the fur transcript was characterized by a noncoding region of $\sim 1.6 \mathrm{~kb}(1,2)$. Furthermore, it appeared from analyses of a $3.1-\mathrm{kbp}$ cDNA clone that fur contained an open reading frame of at least $1,500 \mathrm{bp}$. In the amino acid sequence deduced from it, a hydrophobic region was present at a position of $\sim 50$ amino acid residues from the

Address reprint requests to Dr. W. J. M. Van de Ven, Molecular Oncology Section, Department of Biochemistry, University of Nijmegen, Geert Grooteplein Noord 21, 6525 EZ Nijmegen, The Netherlands.

Received for publication 15 January 1987 and in revised form 22 April 1987.

J. Clin. Invest.

(c) The American Society for Clinical Investigation, Inc.

$0021-9738 / 87 / 12 / 1545 / 05 \$ 2.00$

Volume 80, December 1987, 1545-1549 carboxy terminus. This potential transmembrane domain was very similar to the ones found in some proteins encoded by class II genes of the MHC. Upstream of the transmembrane domain, a cysteine-rich region was present. Cysteine-rich regions appear widely distributed among receptor proteins and, in some cases, may be involved in ligand binding; they are found in the human insulin receptor (3), the human epidermal growth factor receptor (4), the glucocorticoid receptor (5), the oestrogen receptor $(4,6)$, and the LDL receptor (7). Significant homology, especially with respect to the topography of cysteine residues, was found between the cysteine-rich regions of human epidermal growth factor receptor, human insulin receptor, and furin, the putative translational product of fur (2). A transmembrane domain adjacent to a cysteine-rich region, as in furin, is more often observed in receptors (3-8). The characteristics described above make furin a likely candidate for a receptor for an as yet unknown ligand.

Because of the structural properties of furin, it was of interest to study the expression pattern of fur in normal and neoplastic tissue. In the present report, we describe the results of such a study in which we used poly(A)-selected RNA from specimens of a number of different organs of African green monkey and rat, as well as from normal lung tissue that was free of neoplastic lesions and from human lung carcinomas of different histopathological types.

\section{Methods}

Lung carcinomas. Lung carcinoma specimens from 49 patients were selected from the files of the Pathology Department of the St. Antonius Hospital, Nieuwegein, The Netherlands. Surgical specimens were presented fresh and sterile to the pathologist immediately after extirpation, and tumor tissue was quickly frozen in liquid nitrogen and stored at $-70^{\circ} \mathrm{C}$. Tumors were characterized by routine microscopical and histopathological techniques and classified according to the criteria of the World Health Organization (9) as small cell lung carcinoma $(\mathrm{SCLC})^{1}$ ( 9 cases) or nonsmall cell lung carcinoma (NSCLC) ( 40 cases). All SCLCs were examined by electron microscopy to confirm the presence of neuro endocrine granules. The NSCLCs could be classified as squamous cell carcinomas (19 cases) and adenocarcinomas (21 cases).

Specimens of normal tissue. Specimens of normal tissue were obtained from cat, rat, mouse, and monkey. Specimens were quickly frozen in liquid nitrogen immediately upon removal from the animal and stored at $-70^{\circ} \mathrm{C}$.

DNA probes and hybridization. DNA probes were isolated and labeled as described (10). To study fur expression, a 3.1-kbp fur-specific cDNA probe was used that was isolated from an oligo (dT)-primed

1. Abbreviations used in this paper: SCLC, small cell lung carcinoma; NSCLC, non-SCLC. 

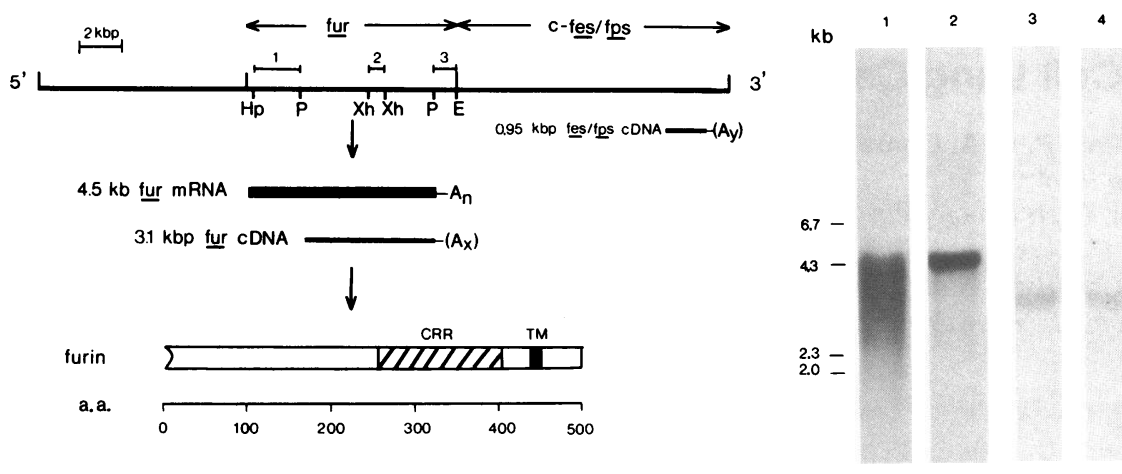

Figure 1. Schematic representation of characteristics of fur. At the top of the figure, the position of fur relative to the $f e s / f p s$ proto-oncogene in the human genome is depicted. The three numbered bars represent fur-related genomic probes. The 4.5-kb fur mRNA is shown below the human genomic region followed by a 3.1-kb fur cDNA clone, which was used as a probe. At the bottom of the figure, the deduced fur translational product is represented as a bar. Under the bar representing the genomic DNA of the proto-oncogene a $0.95-\mathrm{kbp}$ fes/ fps cDNA clone (3) is shown. At the right of the figure, a Northern blot analysis of

poly(A)-selected RNA from KG-1 cells is shown. Probes included a combination of genomic probes 1, 2, and 3 (lane 1), the 3.1-kbp fur cDNA clone (lane 2), the 0.95-kbp fes/ fps cDNA clone (lane 3), and the v-fes-specific $S_{1}$ probe (16) (lane 4). Molecular weight markers include $\lambda$-DNA digested with restriction endonuclease Hind III. CRR, cysteine-rich region; TM, transmembrane domain; E, Eco RI; Hp, Hpa I; P, Pst I; Xh, Xho I.

cDNA library (2) from KG-1 cells (11). The 0.95-kbp fes/fps-specific cDNA probe was described before (1) and included the genetic sequences of the last five exons of c-fes/fps (12). To assay the presence of actin transcripts, pAct-1 a hybrid molecule of pBR322 and $1.2 \mathrm{kbp}$ of actin-specific cDNA sequences of hamster origin (13) was used. Hybridization experiments on nitrocellulose membranes were performed as described previously (10). For hybridizations on nylon membranes (Hybond-N; Amersham Corp., Arlington Heights, IL) the method of Church and Gilbert (14) was used. The nylon membranes were dehybridized by incubation in $5 \mathrm{mM}$ Tris- $\mathrm{HCl}$ (pH 8.0), $2 \mathrm{mM}$ EDTA and $0.1 \times$ Denhardt's solution ( $1 \times$ Denhardt's solution contains $0.02 \%$ (wt/vol) BSA, $0.02 \%(\mathrm{wt} / \mathrm{vol})$ polyvinylpyrrolidone and $0.02 \%(\mathrm{wt} / \mathrm{vol})$ ficoll at $65^{\circ} \mathrm{C}$ for $2 \mathrm{~h}$. Each dehybridization was checked by autoradiography. Blots could be used several times without significant loss of signal.

mRNA isolation and Northern blot analysis. Total cellular RNA was isolated from normal and malignant lung tissue using the lithium-urea procedure described by Auffray and Rougeon (15). $10 \mu \mathrm{g}$ of oligo (dT)-cellulose purified mRNA was glyoxalated and size fractionated on $1.0 \%$ agarose gels and transferred to Hybond-N (procedure as recommended by Amersham Corp.).

\section{Results}

Differential expression of fur in normal tissues of monkey and rat. We have studied the expression pattern of the recently discovered fur gene in normal tissues of different histological types by Northern blot analysis. For this study, a suitable furspecific probe was selected. In Fig. 1, characteristics of the fur gene are summarized. With a combination of genomic probes representing a major portion of the fur transcription unit (probes 1, 2, and 3; see Fig. 1) the 4.5-kb fur transcript could be detected in poly(A)-selected RNA from the human cell line KG-1. However, a smear of transcripts of lower molecular weight was always observed (Fig. 1, lane 1). When a 3.1-kbp fur-specific cDNA clone (Fig. 1) was used, a clear and distinct band was obtained instead (Fig. 1, lane 2). As can be seen in Fig. 1, this cDNA clone represented about $70 \%$ of the $4.5-\mathrm{kbp}$ fur transcript and contained the genetic sequences that encode the transmembrane domain and the cysteine-rich region. The smearing effect observed with the combined genomic probes was probably due to repetitive sequences most likely located in the $5^{\prime}$ region of fur (data not shown). To study the fes/fps transcripts, a 0.95-kbp fes/ fps-specific cDNA was used (Fig. 1, lane 3) instead of the v-fes-specific $S_{1}$ probe (16) (Fig. 1, lane 4).
The facts that the fur cDNA probe was of human origin as well as that the availability of specimens from a number of different human organs was rather limited led us to test the evolutionary conservation of the fur gene to select an appropriate alternative. This was performed by Northern blot analysis of poly(A)-selected RNA from kidney specimens of mouse, monkey, man, rat, and cat (Fig. 2). Although some variation in the signal strength could be observed, the fur gene seemed fairly well conserved during evolution and could be detected in all these species under hybridization conditions of high stringency. The mRNA of rat appeared a little shorter as could be observed upon prolonged agarose gel electrophoresis (data not shown). Based on these observations, we chose to test fur expression in various tissues of African green monkey and rat. In an attempt to compare similar amounts of poly(A)-selected RNA, we used $\mathrm{OD}_{260}$ and ethidium bromide staining to estimate RNA concentrations. Results of Northern blot analysis of poly(A)-selected RNA from a number of tissues of African green monkey are summarized in Fig. 3. Relatively high levels of fur transcripts were found in RNA from liver and kidney. Lower levels of fur expression were observed in brain, spleen, and thymus, and very low levels in heart muscle, lung, and testis. Expression of fur was also found in some established cell lines (data not shown), for instance in KG-1 cells (Fig. 1, lanes

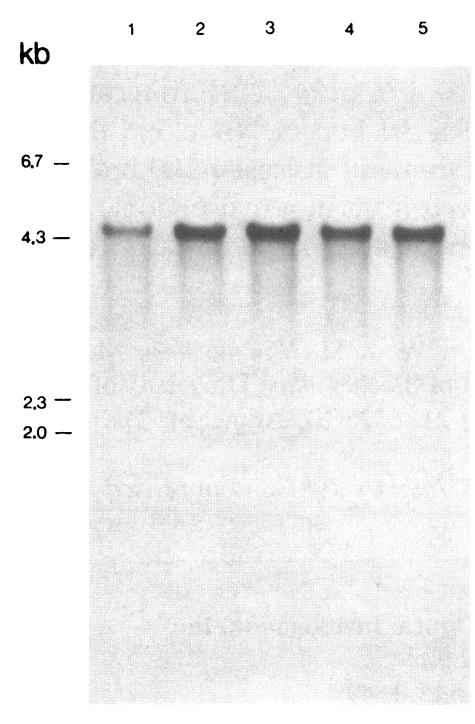

Figure 2. Northern blot analysis of poly(A)-selected RNA (20 $\mu \mathrm{g}$ amounts) isolated from kidney of mouse (lane 1), monkey (lane 2), man (lane 3), rat (lane 4), and cat (lane 5). Molecular weight markers are the same as those described in the legend to Fig. 1. 
A

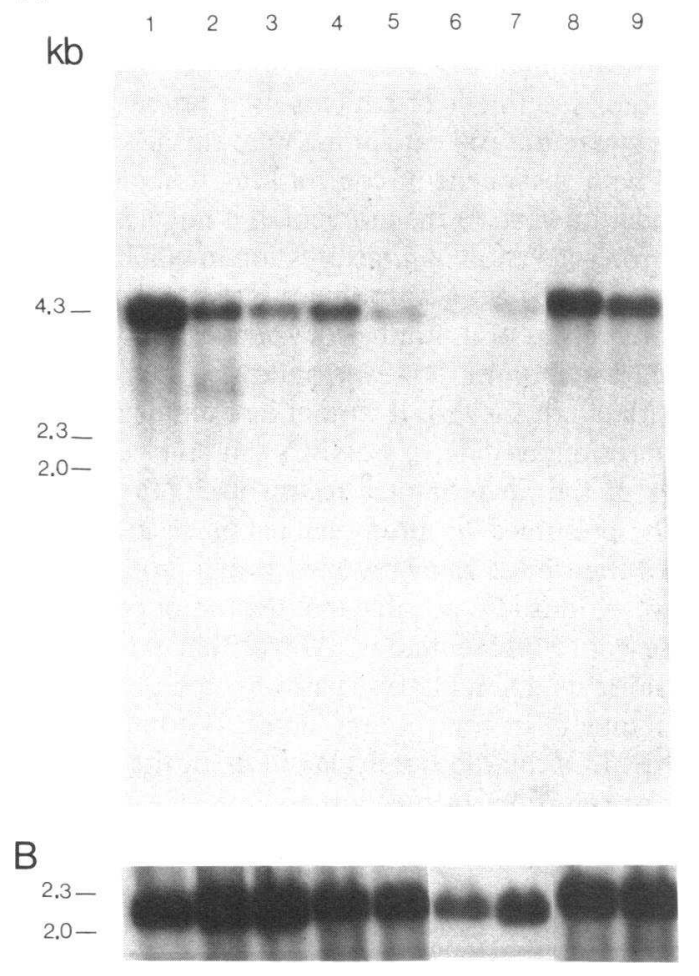

Figure 3. Expression of fur and fes/fps in a number of tissues of an African green monkey and Hela cells. $(A)$ Northern blot analysis was performed with poly(A)-selected RNA (20 $\mu \mathrm{g}$ amounts) of liver (lane 1), spleen (lane 2), brain (lane 3), thymus (lane 4), lung (lane 5), testis (lane 6), heart muscle (lane 7), kidney (lane 8), and Hela cells (lane 9). As a molecular probe, the 3.1-kbp fur cDNA was used. Upon autoradiography, the blot was screened with the $0.95-\mathrm{kb}$ fes/ fps cDNA. Molecular weight markers are the same as those described in the legend to Fig. 1. (B) The same blot as that used in part $A$ of the figure was used in a Northern blot analysis with an actinspecific probe.

1 and 2) and in Hela cells (Fig. 3, lane 9). It should be emphasized that the autoradiograph shown in Fig. $3 \mathrm{~A}$ was exposed $24 \mathrm{~h}$ (using Kodak XAR-5 and two intensifying screens [Lightning Plus; DuPont Photo Products, Burbank, CA]). In Fig. 3 $B$, levels of actin transcripts in the various samples are shown (hybridization of the same Northern blot) as an additional control experiment. Exposure in this experiment was for $4 \mathrm{~h}$. A similar expression pattern of fur was observed with RNA from various rat tissues (data not shown). These experiments indicate that the fur gene exhibits a differential expression pattern. At present, it is not clear whether the weak hybridization signals observed with specimens of some organs were due to low levels of fur transcripts in the tissue-specific cells of these specimens or to the presence of cells of hemopoietic origin that exhibit relatively higher levels of fur expression. In situ hybridization or immunofluorescence analysis could resolve this matter and establish in detail which cell types contain the fur transcripts or its translational product.

When the same blots were assayed for the presence of fes/ $f p s$-related sequences without prior dehybridization, a hybridization signal at $3.0 \mathrm{~kb}$ was observed only in spleen samples (Fig. $3 A$, lane 2). This observation is in agreement with previous reports indicating expression of $f e s / f p s$ to be restricted mainly to hemopoietic cells of the myeloid lineage (17-21; see also Fig. 1, lanes 3 and 4 ). Note that in Fig. 3 the fur-specific and the $f e s / f p s$-specific hybridization are superimposed, in which the fur-specific signal is the result of the first hybridization.

Expression of fur in human lung carcinomas. The observation that fur is expressed at very low levels in tissues like heart muscle, lung, and testis led us to extend our studies to naturally occurring tumors. Because of its high incidence in man, lung carcinomas were an obvious first choice for such a study.

We have studied nine cases of SCLC and 40 cases of NSCLC. The latter included 19 cases of squamous lung cell carcinoma and 21 cases of adenocarcinoma. For control experiments we used specimens of lung tissue without neoplastic lesions from man, cat, rat, and monkey. In Fig. $4 \mathrm{~A}$, the results of a Northern blot analysis of similar amounts of poly(A)-selected RNA from the various types of lung carcinomas and the various control lung tissues are presented. Note that conditions were the same in all experiments and that exposure of the film was $10 \mathrm{~h}$ (using Kodak XAR-5 and two intensifying screens [Lightning Plus; DuPont Photo Products]). Normal lung tissue of man (lane 1), cat (lane 2), rat (lane 3), and monkey (lane 4) exhibited very low levels of fur expression (see also Fig. 3). Similarly, in SCLCs fur expression was also just above the detection level under these conditions. In the NSCLCs, however, strongly elevated levels of fur transcripts were observed (lanes 7-10). We estimated these to be $\sim 10$ to 25 times higher than that in control lung tissue or SCLCs and $\sim$ five- to tenfold higher than that in liver and kidney. No major differences in the levels of fur expression in squamous cell carcinomas and adenocarcinomas could be observed. In Fig. $4 B$, the actin hybridization pattern of the same blot as

A

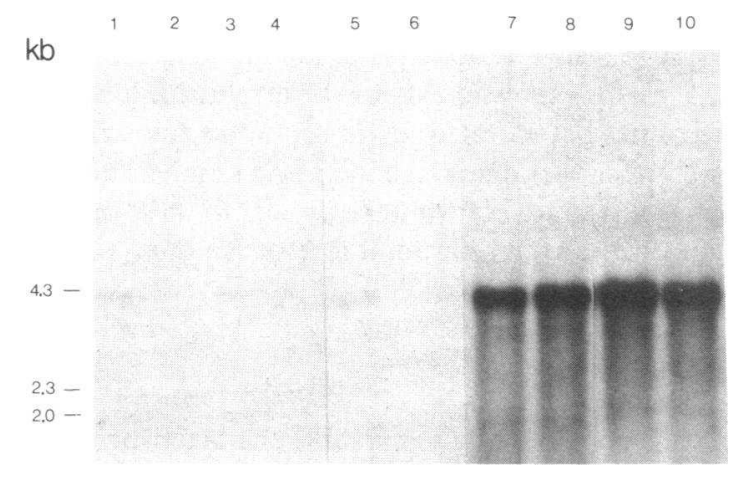

B

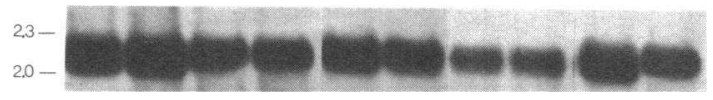

Figure 4. Expression of fur in normal and neoplastic lung tissue. $(A)$ Northern blot analysis was performed with poly(A)-selected RNA (20 $\mu \mathrm{g}$ amounts) of normal lung tissue of man (lane 1 ), cat (lane 2), rat (lane 3), and monkey (lane 4), plus of two human SCLCs (lanes 5 and 6), two human adenocarcinomas (lanes 7 and 8 ), and two human squamous cell carcinomas (lanes 9 and 10). As a molecular probe, the 3.1-kbp fur cDNA was used. $(B)$ Control for the amounts of RNA on Hybond-N paper. The same blot as that used in part $A$ of the figure was used in an hybridization analysis with an actin-specific probe. 
Table I. Fur Gene Expression in Human Lung Carcinoma Specimens

\begin{tabular}{|c|c|c|c|c|}
\hline \multirow[b]{2}{*}{ Human lung tissue } & \multirow{2}{*}{$\begin{array}{l}\text { Total numbers of } \\
\text { specimens tested }\end{array}$} & \multicolumn{3}{|c|}{$\begin{array}{l}\text { Numbers in different } \\
\text { categories of fur } \\
\text { expression }\end{array}$} \\
\hline & & $++^{*}$ & $+^{\ddagger}$ & Control \\
\hline SCLC & 9 & 0 & 1 & 8 \\
\hline Squamous cell carcinoma & 19 & 14 & 2 & 3 \\
\hline Adenocarcinoma & 21 & 18 & 2 & 1 \\
\hline Control lung & 5 & - & - & 5 \\
\hline
\end{tabular}

* ++ Indicates a level of fur expression of 10 to 25 times higher than the level observed in control lung tissue specimens, as determined by densitometric measurements.

$\ddagger$ Indicates a level of fur expression of $\sim$ five times higher than that observed in control lung tissue specimens, as determined by densitometric measurements.

used in part $A$ is presented to indicate the relative amounts of actin mRNA on the blot. In Table I, the results of all lung carcinomas are summarized. In most NSCLC cases, levels of fur expression were 10 to 25 times higher than that in specimens of control lung tissue. In four cases, fur mRNA levels were $\sim$ five times higher than control levels and in four other cases no elevation of fur transcription was observed. In the specimen of one of the nine SCLC patients, fur levels were elevated to some extent. The eight others were all very low.

The same Northern blots were also used to study expression of the fes/fps proto-oncogene. Expression of the protooncogene appeared to be low in all lung tissues tested, although some elevation of expression could be observed in some of the lung tumors tested (data not shown). The presence of low levels of $f e s / f p s$ in lung tissue is already reported by others (17-21). However, it is not clear at the moment to which cell types the fes/fps expression should be attributed. It is possible that the somewhat elevated levels of $f e s / f p s$ transcription in lung carcinomas was due to the increased numbers of alveolar macrophages that are known to be present in these tumors. In situ hybridization or immunofluorescence analysis should clarify this issue.

\section{Discussion}

The fur gene possesses two remarkable characteristics. First of all, its receptor-like structural features, the presence of a transmembrane domain and a cysteine-rich region, and, secondly, its evolutionary conserved location in the region immediately upstream of the fes/ fps proto-oncogene. The fact that genetic sequences with coding potential for a protein with receptorlike characteristics remained closely linked to a proto-oncogene that encodes a tyrosine- specific protein kinase is interesting since it raises the possibility of a functional relationship. The expression pattern of the $f e s / f p s$ proto-oncogene is known to be restricted to the myeloid lineage of hemopoietic cells (21). Analysis of the expression pattern of fur could, therefore, provide insight in a potential relationship between fur and the proto-oncogene. The differential expression pattern of fur described in this paper, however, shows differences in regulation of expression of fur and fes/ fps implicating a functional role of the fur gene product that is not necessarily linked to the protooncogene product.

The observation that expression levels of fur transcripts in most lung tumor specimens of patients with primary adenocarcinoma or squamous cell carcinoma were strongly elevated as compared with specimens of control lung tissue or SCLC was of particular interest. At the moment, it is not clear how to explain the strong elevation of fur expression in NSCLCs. As a possible explanation, gene amplification was considered. Southern blot analysis of a number of adenocarcinomas and squamous cell carcinomas, however, indicated that this was not the case (data not shown). It cannot be excluded that the enhanced transcription of fur in NSCLCs should be explained by properties of the fur-promoter region itself. Preliminary analysis of the presumed fur-promoter region in a chloramphenicolacetyl transferase assay revealed strong promoter-like activity. However, it is also possible that the tumor cells in the NSCLCs arise from a differentiating cell type that expresses fur somewhere along its differentiating pathway and at the same time is low abundant in control lung tissue. Becoming abundant in the NSCLCs could be an explanation for the observed increase in fur expression. In such a model, the few cases that did not exhibit elevation of fur expression could also be explained.

Resected SCLC specimens are very uncommon, representing the minority of all cases. Thus, SCLCs amenable to surgical resection may be biologically different from SCLCs present in the standard unresectable manner. With respect to fur gene expression, we did not see such a biological difference in the nine SCLC cases we studied. Only one of them exhibited a slightly elevated level of fur transcripts. Of the nine SCLC specimens, four were obtained by surgical resection. Diagnosis of these four cases was based on histology, cytology, immunohistochemistry, and electron microscopy. Survival rates of the patients varied between 6 and 24 mo (average, $12.7 \mathrm{mo}$ ). Tumor specimens of the remaining five cases were obtained during autopsies that were performed within $1 \mathrm{~h}$ of the patient's death. Diagnosis of one of these cases was based on histology and immunohistochemistry (survival, $3 \mathrm{mo}$ ). Diagnosis of the remaining four cases was based on histology only. Survival rates of these four cases varied between 3 wk and 8 mo (average, $3 \mathrm{mo}$ ). In accordance with the diagnoses, all nine cases exhibited a biological behavior of small cell undifferentiated lung carcinoma.

The differential expression pattern of fur in SCLC and NSCLC also implied that fur gene expression could be used as a discriminating marker in studies on human lung cancer. At present, SCLC cells can be identified using a number of biomarkers (for a review see reference 22). For example, gastrinreleasing peptide is shown to be a suitable marker for SCLC cells (23). A similarly useful marker for NSCLCs, which account for $\sim 75-80 \%$ of all cases of primary lung cancer, is not available at present (22). The identification of fur as a potential marker for NSCLCs could, therefore, be of importance for lung cancer diagnosis. Analysis of the fur gene has so far $(1,2)$ revealed promising characteristics of the gene and its product. First of all, evidence that furin represents a cell surface receptor is in favor of relatively easy accessibility of the protein. Moreover, the fact that in NSCLCs the levels of fur transcripts are selectively and strongly elevated may further facilitate furin detection. Both these characteristics could make the fur 
gene a valuable object for the development of reagents to detect NSCLC in an early stage.

\section{Acknowledgments}

We thank M. Bussemakers and A. ter Avest for their contributions to this study and E. Vaarkamp for preparing tumor tissue specimens. We thank A. van den Ouweland for helpful comments.

This work was supported by the Netherlands Cancer Foundation, contract NUKC Bioch. 85-9.

\section{References}

1. Roebroek, A. J. M., J. A. Schalken, M. J. G. Bussemakers, H. van Heerikhuizen, C. Onnekink, F. M. J. Debruyne, H. P. J. Bloemers, and W. J. M. Van de Ven. 1986. Characterization of human c-fes/ fps reveals a new transcription unit ( $f u r)$ in the immediately upstream region of the proto-oncogene. Mol. Biol. Rep. 11:117-125.

2. Roebroek, A. J. M., J. A. Schalken, J. A. M. Leunissen, C. Onnekink, H. P. J. Bloemers, and W. J. M. Van de Ven. 1986. Evolutionary conserved close linkage of the c-fes/fps proto-oncogene and genetic sequences encoding a receptor-like protein. EMBO (Eur. Mol. Biol. Organ.) J. 5:2197-2203.

3. Ullrich, A., J. R. Bell, E. Y. Chen, R. Herrera, L. M. Petruzelli, T. J. Dull, A. Gray, L. Coussens, Y.-C. Liao, M. Tsubokawa, A. Mason, P. H. Seeburg, C. Grunfeld, O. M. Rosen, and J. Ramachandran. 1985. Human insulin receptor and its relation to the tyrosine kinase family of oncogenes. Nature (Lond.). 313:756-761.

4. Ullrich, A., L. Coussens, J. S. Hayflick, T. J. Dull, A. Gray, A. W. Tam, J. Lee, Y. Yarden, T. A. Libermann, J. Schlessinger, J. Downward, E. L. V. Mayes, N. Whittle, M. D. Waterfield, and P. H. Seeburg. 1984. Human epidermal growth factor receptor $\mathrm{CDNA}$ sequences and aberrant expression of the amplified gene in A431 epidermoid carcinoma cells. Nature (Lond.). 309:418-425.

5. Hollenberg, S. M., C. Wienberger, E. S. Ong, G. Cerelli, A. Oro, E. B. Thompson, M. G. Rosenfeld, and R. M. Evans. 1985. Primary structure and expression of a functional human glucocorticoid receptor cDNA. Nature (Lond.). 318:635-641.

6. Green, S., P. Walter, V. Kunar, A. Krust, J. Bornhert, P. Argos, and P. Chambon. 1986. Human oestrogen receptor cDNA: sequence, expression and homology to v-erb-A. Nature (Lond.). 320:134-139.

7. Südhof, T. C., J. L. Goldstein, M. S. Brown, and D. W. Russell. 1985. The LDL receptor gene: a mosaic of exons shared with different proteins. Science (Wash. DC). 228:815-822.

8. Yamamoto, T., C. G. Davis, M. S. Brown, W. J. Schneider, M. L. Casey, J. L. Goldstein, and D. W. Russell. 1984. The human LDL receptor: a cysteine-rich protein with multiple Alu sequences in its mRNA. Cell. 39:27-38.

9. World Health Organization. 1982. The world health organization histological typing of lung tumors. Am. J. Clin. Pathol. 77:123136.
10. Van den Ouweland, A. M. W., M. L. Breuer, P. H. Steenbergh, J. A. Schalken, H. P. J. Bloemers, and W. J. M. Van de Ven. 1985. Comparitive analysis of the human and feline c-sis proto-oncogenes. Identification of 5 ' human c-sis coding sequences that are not homologous to the transforming gene of simian sarcoma virus. Biochim. Biophys. Acta. 825:140-147.

11. Koeffler, H. P., and D. W. Golde. 1978. Acute myelogenous leukemia: a human cell line responsive to colony-stimulating activity. Science (Wash. DC). 200:1153-1154.

12. Roebroek, A. J. M., J. A. Schalken, J. S. Verbeek, A. M. W. Van den Ouweland, C. Onnekink, H. P. J. Bloemers, and W. J. M. Van de Ven. 1985. The structure of the human c-fes/fps proto-oncogene. EMBO (Eur. Mol. Biol. Organ.) J. 4:2897-2903.

13. Dodemont, H. J., P. Soriano, W. J. Quax, F. Ramaekers, J. A. Lenstra, M. A. M. Groenen, G. Bernardi, and H. Bloemendal. 1982. The genes coding for the cytoskeletal proteins actin and vimentin in warm-blooded vertebrates. EMBO (Eur. Mol. Biol. Organ.) J. 1:167171.

14. Church, G. M., and W. Gilbert. 1984. Genomic sequencing. Proc. Natl. Acad. Sci. USA. 81:1991-1995.

15. Auffray, Ch., and F. Rougeon. 1980. Purification of mouse immunoglobulin heavy-chain messenger RNAs from total myeloma tumor RNA. Eur. J. Biochem. 107:303-314.

16. Franchini, G., J. Even, C. J. Sherr, and F. Wong-Staal. 1981. onc sequences ( $\mathrm{v}-\mathrm{fes}$ ) of Snyder-Theilen feline sarcoma virus are derived from noncontiguous regions of a cat cellular gene (c-fes). Nature (Lond.). 290:154-157.

17. Barbacid, M., K. Beemon, and S. G. Devare. 1980. Origin and functional properties of the major gene product of the Snyder-Theilen strain of feline sarcoma virus. Proc. Natl. Acad. Sci. USA. 77:51585162.

18. Feldman, R. A., J. L. Gabrilove, J. P. Tam, M. A. S. Moore, and $H$. Hanafusa. 1985. Specific expression of the human cellular fps/ fes-encoded protein NCP92 in normal and leukemic myeloid cells. Proc. Natl. Acad. Sci. USA. 82:2379-2383.

19. Mathey-Prevot, B., H. Hanafusa, and S. Kawai. 1982. A cellular protein is immunologically cross reactive with and functionally homologous to the Fujinami sarcoma virus transforming protein. Cell. 28:897-906.

20. McDonald, I., J. Levy, and T. Pawson. 1985. Expression of the mammalian c-fes protein in hematopoietic cells and identification of a distinct fes-related protein. Mol. Cell. Biol. 5:2543-2551.

21. Slamon, D. J., J. B. deKernion, I. M. Verma, and M. J. Cline. 1984. Expression of cellular oncogenes in tumors. Science (Wash. DC). 224:256-262.

22. Minna, J. D., G. A. Higgins, and E. J. Glatstein. 1985. Cancer of the lung. In Cancer Principles and Practice of Oncology. 2nd ed. V. T. Devita, Jr., S. Hellman, and S. A. Rosenberg, editors. J. B. Lippincott Co., Philadelphia. 507-597.

23. Sausville, E. A., A.-M. Lebacq-Verheyden, E. R. Spindel, F. Cuttitta, A. F. Gazdar, and J. F. Battey. 1986. Expression of the gastrin-releasing peptide gene in human small cell lung cancer. J. Biol. Chem. 261:2451-2457. 